\title{
Abstracts from Frontiers Research Day University of Kansas Medical Center, Kansas City, KS March 4, 2020
}

31 Racial Disparities in Cesarean Delivery in Singleton Pregnancies Jenifer Allsworth, Ph.D.

32 The Chaning Health and Social Circumstances of Women Leaving Jails: A Three-Year Longitudinal Study Stephanie Assimonye, B.S., Jaehoon Lee, Ph.D., Sharla Smith, Ph.D., MPH, Jason Glenn, Ph.D., Megha Ramaswarmy, Ph.D., MPH

33 Estimation and Construction of Confidence Intervals for the Cutoff-Points of Continuous Biomarkers Under the Euclidean Distance in 3D Settings

Brian Mosier, B.S., Leonidas Bantis, Ph.D.

34 Humanized Mouse Models for Studying the Role of Immune Cells in Breast Cancer Progression Hanan Elsarraj, Carolyn Kafuman, Darlene Limback, Yan Hong, Haleigh Harper, Lawrence R. Ricci, Fang Fan, Ossama Tawfik, Lisa May, Therese Cusick, Marc Inciardi, Mark Redick, Jason Gatewood, Onalisa Winblad, Tim Fields, Carol Fabian, Andrew K. Godwin, Patrick E. Fields, Ruby Meierotto, Linheng Li, John Perry, Fariba Behbod

35 Evaluating the Adverse Effects of Hyperglycemia on the Airway Epithelium in Cystic Fibrosis and CF-related Diabetes Charles Bengston, M.D.

36 University of Kansas Medical Center Genomics Core Clark Bloomer, B.S.

37 Analysis of Mitochondrial Haplogroups with Alzheimer Disease Risk Palash Sharma, M.S., Jonathan Mahnken, Ph.D., Dongwei Hui, Ph.D., Mary Michaelis, Ph.D., Elias Michaelis, M.D., Ph.D., Russell Swerdlow, M.D., Prabhakar Chalise, Ph.D.

38 Live Cell and Intravital Imaging Reveals Differences in Mitochondrial Redox State, Morphology and Number between Osteoblasts and Osteocytes

Sarah Dallas, Ph.D.

39 Targeting Prolactin Receptor Using FDA-Approved Drugs: A Molecular Docking Approach and Drug Binding Studies Prasad Dandawate, Ph.D.

40 Neural Predictors of "Rapid Response" in Anorexia Nervosa Victoria Perko, Kelsi Forbush, Ph.D., Sara Gould, Brianne Richson, Kylie Christian, Danielle Chapa, Laura Martin

41 A High Throughput Assay for Quantitating $\beta$-catenin Signaling in the MLO-Y4 Osteocyte-like Cell Line Mark Johnson, Ph.D.

42 Exploratory Evaluation of an Online Educational Intervention for JUUL Use Matthew J. Carpenter, Ph.D., Tracy T. Smith, Ph.D., Nikki Nollen, Ph.D., Eleanor Leavens, Ph.D.

43 American Indian College Students' Knowledge, Attitudes, Beliefs, and Behaviors Surrounding Alternative Tobacco Products

Charley Lewis, MPH

44 Low Dose Daunorubicin Targets the Leukemia Stem Cell in AML

Tara L. Lin, M.D., John M. Perry, Ph.D., Xi He, Ph.D., Gregory Reed, Ph.D., Na Zhang, Ph.D.,

Scott Weir, Pharm.D., Joseph P. McGuirk, D.O., Linheng Li, Ph.D. 


\section{Abstracts from Frontiers Research Day University of Kansas Medical Center, Kansas City, KS March 4, 2020}

45 In Our Sacred Voice: An Exploration of Tribal and Community Leader Perceptions as Health Communicators of Disease Prevention among American Indians in the Plains

Crystal Y. Lumpkins, Ph.D., M.A., Ryan Goeckner, M.A., Jason Hale, M.A., Charley Lewis, MPH,

Jordyn Gunville, MPH, River Gunville, B.A., Chris M. Daley, Ph.D., M.A.

46 Not About Us, Without Us: An Exploratory Study of Underserved and Minority Patients' Views of CancerRelated Genetic Counseling and Testing Communication

Crystal Y. Lumpkins, Ph.D., M.A., Alisdair Philp, Ph.D., M.S., CGC, Kim Kimminau, Ph.D.,

Mariana Ramirez-Mantilla, LMSW, Reem Mustafa, MBBS, Ph.D., MPH, Andrew Godwin, Ph.D.,

Yani Vazquez, MBA, Nancy Washington, Kim Jones, Shaton Freeman

47 Changing how the Brain Responds when Making Decisions: Translating Neuroscience to Population Health Laura E. Martin, Ph.D., Morgan G. Brucks, B.A., Andrew Fox, Ph.D., Vlad B. Papa, B.A.,

Noreen D. Mdege, Ph.D., MPH, Austin S. Baldwin, Ph.D., Elisa M. Trucco, Ph.D., Nina A. Cooperman, Psy.D., Angelos P. Kassianos, Ph.D., Sara M. Levens, Ph.D.

48 Engaging Patients and Defining Outcomes: A Minority Engagement Effort Reem Mustafa, M.D., MPH, Ph.D.

49 Race-Related Stress and Food Decision Making in African American Women Ebony Onianwa, B.S.

50 Low Socioeconomic Status and Residential Distance of Less than 10 Miles from a Frontier-State $\mathrm{NCl}$-Designated Cancer Center is Associated with Worse Ovarian Cancer Survival Shariska Petersen, M.D.

51 Understanding Race Bias in the Decision to Shoot with an Integrated Model of Decision Making Timothy J. Pleskac, Ph.D., Sergej Grunevski, B.S., Taosheng Liu, Ph.D., Joseph Cesario, Ph.D.

52 A Developing Shared Resource: Emerging Imaging Technologies and Applications Laboratory Eduardo Rosa-Molinar, Ph.D.

53 Influence of Vision and Proprioception on Motor Control in ASD Robin L. Shafer, Ph.D., Zheng Wang, Ph.D., Matthew W. Mosconi, Ph.D.

54 Health through Enhancing Awareness and Learning about Breast and Cervical Cancer Screening among African American Women (HEAL)

Sharla Smith, MPH, Ph.D., Jannette Berkley-Patton, Ph.D., Megha Ramaswamy, MPH, Ph.D., Joi Wickliffe, MPH

55 RITUXimab IMmunogenicity in ANCA-associated Vasculitis (RITUXIMAV)

Jason Springer, M.D., M.S., Ryan Funk, Pharm.D., Ph.D.

56 Open-Label Pilot Study of Ranolazine for Cramps in Amyotrophic Lateral Sclerosis Jeffrey Statland, M.D.

57 Skin Carotenoid Measurement: A Biomarker for Dietary Fruit and Vegetable Intake Debra Sullivan, Ph.D., RD

58 Cognitive Function and Relationships with Intervention Dropout, Adherence and Weight Loss Amanda Szabo-Reed, Ph.D.

59 Adolescent Mothers' Early Breastfeeding Experiences Karen Wambach, Ph.D., RN, IBCLC, FILCA, FAAN

60 A Therapeutic Trial Validating the Physiologically Based Pharmacokinetic Simulation of the Dose-Exposure Relationship of Metformin in Young Children Karen Wambach, Ph.D., RN, IBCLC, FILCA, FAAN 


\section{Abstracts from Frontiers Research Day University of Kansas Medical Center, Kansas City, KS March 4, 2020}

61 A Therapeutic Trial Validating the Physiologically Based Pharmacokinetic Simulation of the Dose-Exposure Relationship of Metformin in Young Children with Insulin Resistance or Type 2 Diabetes Mellitus Chelsea Cojocari, Ph.D., Rachel Frazier, RN, BSN, CCRC, Kelsee Halpin, M.D., Paul Toren, Ph.D., J. Steven Leeder, Pharm.D., Ph.D., Yun Yan, M.D.

62 Targeting RNA-Binding Protein HuR in Human Liver Cancer

Priyanka Ghosh, Lin He, Nancy Magee, Forkan Ahamed, Xiaoqing Wu, Liang Xu, Yuxia Zhang 


\title{
KANSAS JOURNAL of MEDICINE
}

\author{
Jenifer Allsworth, Ph.D. \\ Associate Professor \\ Department(s) of Biomedical and Health Informatics \\ University of Missouri-Kansas City
}

Frontiers Clinical and Translational Pilot Research Grant Recipient

\section{Racial Disparities in Cesarean Delivery in Singleton Pregnancies}

Received Feb. 7, 2020; Accepted for publication Feb. 10, 2020; Published online Feb. 26, 2020

\begin{abstract}
Introduction: The past two decades have seen a revolution in the management of labor and delivery; rates of cesarean delivery (CD), medical induction, and electronic fetal monitoring have increased dramatically. CD occurs in one third of deliveries and is the most common inpatient surgery. The increase in CD has occurred despite concerns about maternal complications (infection, blood loss, hysterectomy, uterine rupture) and racial disparities. Since 1993, black and white women have gone from comparable CD rates to rates $40 \%$ higher among black women compared to white women.
\end{abstract}

Methods: This project has assembled a cohort of over 800,000 deliveries from the Cerner Corporation's Health Facts ${ }^{\circledast}$ database. Health Facts contains 63 million unique patients from over 600 U.S. healthcare facilities. Data include comprehensive clinical records from inpatient and outpatient encounters and includes data from pharmacy, laboratory, registration and billing data.

Results: CD was identified using a validated definition that incorporates International Classification of Disease (Version 9, Clinical Modification) codes for delivery outcome and relevant diagnosis-related groups and procedures. We examined 618,582 singleton deliveries to black and white women and identified 177,564 cesarean deliveries (28.7\%).

Conclusions: The rate of cesarean was higher for black women overall and during the years 2000 - 2006 and 2011 - 2015 the CD rate for black women was significantly higher than for white women. Of note, during the period from 2007 - 2010 rates of cesarean were similar among black and white women. The rate of repeat cesarean was lower among black women, while the rate of induction of labor was higher.

Funding Support: This work was supported by a CTSA grant from NCATS awarded to the University of Kansas for Frontiers: University of Kansas Clinical and Translational Science Institute (\#ULITR002366). The contents are solely the responsibility of the authors and do not necessarily represent the official views of the NIH or NCATS. 
Frontiers Predoctoral Trainee Scholar (TLl)

\section{The Changing Health and Social Circumstances of Women Leaving Jails: A Three-Year Longitudinal Study}

Stephanie Assimonye1, Jaehoon Lee², Ph.D., Sharla Smith³, Ph.D., MPH, Jason Glenn4, Ph.D., Megha Ramaswamy1 , Ph.D., MPH

${ }^{1}$ University of Kansas School of Medicine, Department of Preventative Medicine and Public Health, Kansas City, KS

${ }^{2}$ Texas Tech University, College of Education, Institute for Measurement, Methodology, Analysis, and Policy (IMMAP), Lubbock, TX

${ }^{3}$ University of Kansas School of Medicine-Wichita, Preventive Medicine and Public Health, Wichita, KS

${ }^{4}$ University of Kansas School of Medicine, Department of History and Philosophy of Medicine, Kansas City, KS

Received Feb. 7, 2020; Accepted for publication Feb. 10, 2020; Published online Feb. 26, 2020

\section{ABSTRACT}

Introduction: Ninety-five percent of all inmates are eventually released back into communities, with many being released without access to the most basic of necessities. Women in particular face complex health and social challenges - children, partners, strained employment opportunities, health problems - before incarceration that they must also face upon reentry. This poses a barrier to their successful reintegration back into society.

Methods: The present study analyzes data collected as part of the sexual health empowerment (SHE Project) health literacy intervention. Participants were recruited from three county jails in the greater Kansas City area. At baseline, participants completed a survey that assessed sociodemographic characteristics and social histories prior to incarceration. Women were followed annually after program completion to complete surveys to assess their long-term health and social circumstances. The present study is a secondary analysis of baseline and followup data.

Anticipated Results: We use Hobfoll's Conservation of Resources (COR) Theory to conceptualize the stress that justice-involved women may experience as a result of perceived or actual loss of resources. We hypothesize that "loss spirals", a term coined by Stevan Hobfoll, creates psychological stress that drives justice-involved women to assume risky sex and drug behaviors that will generate more resources and help to cope with the stress.

Significance of Impact: This study aims to define a succinct longitudinal timeline assessing biopsychosocial outcomes of women released from jail in order to improve prevention and intervention techniques for improvement in social and health circumstances of women leaving jail and their reduction in recidivism.

Funding Support: This work was supported by a CTSA grant from NCATS awarded to the University of Kansas for Frontiers: University of Kansas Clinical and Translational Science Institute (\#ULITR002366). The contents are solely the responsibility of the authors and do not necessarily represent the official views of the NIH or NCATS. 


\title{
KANSAS JOURNAL of MEDICINE
}

\section{Leonidas Bantis, Ph.D.}

Assistant Professor

Department of Biostatistics \& Data Science

Biostatistics, Epidemiology and Research Design (BERD) Trailblazer Award Recipient

\section{Estimation and Construction of Confidence Intervals for the Cutoff-Points of Continuous Biomarkers Under the Euclidean Distance in 3D Settings}

Brian Mosier, B.S., Leonidas Bantis, Ph.D.

Received Feb. 7, 2020; Accepted for publication Feb. 10, 2020; Published online Feb. 26, 2020

\begin{abstract}
Pancreatic ductal adenocarcinoma (PDAC) is one of the most lethal forms of cancer with a five-year survival rate $~ 7 \%$. Thus, early detection could be key to reducing its mortality. Finding effective biomarkers is crucial to early detection. Pancreatic cancer biomarker studies typically consider more than two classes (i.e., recruited individuals that are either healthy, suffering from pancreatitis/cysts, or from aggressive PDAC). Conventional ROC curve analysis is the most popular tool to assess the discriminatory ability of biomarkers in distinguishing between two groups. For more than two groups, this approach is inadequate to determine decision cutoffs for decision-making purposes. Currently there is no accuracy-based statistical approach that is immune to the prevalence of the disease and is able to accommodate information from all three groups to optimally determine a decision rule for classification of patients. We propose a Euclidian distance-based approach as a method of estimating optimal cutoff values. We provide a full analysis framework when interest lies in decision-making and provide parametric and nonparametric approaches. Our approaches can easily be generalized into k-class problems when we are interested in distinguishing between four or more classes. We can extend our methods to consider the costs of incorrect decision making for each of the groups and weigh them accordingly. Additionally, combinations of biomarkers or relevant clinical information can also be accommodated straightforwardly to improve decision rules. We illustrate the usefulness of our approaches by analyzing a pancreatic cancer biomarker study conducted at the University of Texas MD Anderson Cancer Center.
\end{abstract}

Funding Support: This work was supported by a CTSA grant from NCATS awarded to the University of Kansas for Frontiers: University of Kansas Clinical and Translational Science Institute (\#UL1TR002366). The contents are solely the responsibility of the authors and do not necessarily represent the official views of the NIH or NCATS. 
Fariba Behbod, Pharm.D., Ph.D.

Professor

Department of Pathology \& Laboratory Medicine

University of Kansas Medical Center

Pathology

CTSA Inter-Institutional Pilot Project Award Recipient

\section{Humanized Mouse Models for Studying the Role of Immune Cells in Breast Cancer Progression}

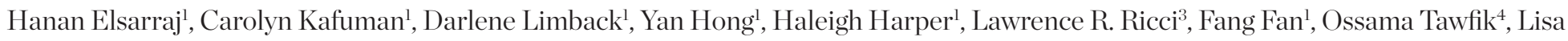

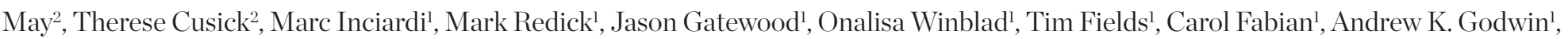
Patrick E. Fields' ${ }^{1}$, Ruby Meierotto ${ }^{4}$, Linheng $\mathrm{Li}^{5}$, John Perry ${ }^{6}$, Fariba Behbod ${ }^{1}$

${ }^{1}$ University of Kansas Medical Center, Kansas City, KS

${ }^{2}$ University of Kansas School of Medicine-Wichita, Wichita, KS

${ }^{3}$ Truman Medical Center, Kansas City, MO

${ }^{4}$ St. Luke's Hospital, Kansas City, MO

${ }^{5}$ Stowers Institute for Medical Research, Kansas City, MO

${ }^{6}$ Children’s Mercy Hospital, Kansas City, MO

Received Feb. 7, 2020; Accepted for publication Feb. 10, 2020; Published online Feb. 26, 2020

\section{ABSTRACT}

Introduction: To study breast cancer progression, we developed the Mouse-Intraductal (MIND) model which involves intraductal injection of breast cancer cells into the mammary ducts of immunocompromised mice, where they display a course remarkably similar to the evolution of human breast cancer, with the formation of lesions in situ followed by invasion into the surrounding stroma. More recently, we are collecting peripheral blood from these same women and have been successful in humanizing mice with the patient's own hematopoietic stem cells (HSC).

Preliminary Data: We have achieved successful engraftment of peripheral blood-derived and bone marrow-derived human CD34+ cells into NSG (immunodeficient NOD-SCID Il2rg-/-), MISTRG (immunodeficient Rag2-/-Il2rg-/- transgene SIRPa expressing GM-CSF, IL-3, M-CSF, TPO) and MISTRG6 mice (plus IL-6) and observed recruitment of their progenitors to breast cancer lesions. Our preliminary studies demonstrate preferential myeloid development when using peripheral blood derived CD $34+$ cells, while both myeloid and lymphoid development are achieved when using bone marrow derived CD34+ cells. Furthermore, MISTRG6 mice show improved development of both human myeloid and lymphoid hematopoietic cells compared to MISTRG and NSG mice.

Impact and Future Aims: The major advantage of our model is the ability to observe progression of patient breast cancer and their associated immune cells in vivo. Analyzing patient samples eliminates the ability to track the progression of lesions and their associated immune cells in vivo. By maximizing these unique advantages, we hope our results will help guide individualized immunotherapy in future patient care.

Funding Support: This work was supported by a CTSA grant from NCATS awarded to the University of Kansas for Frontiers: University of Kansas Clinical and Translational Science Institute (\#ULITR002366). The contents are solely the responsibility of the authors and do not necessarily represent the official views of the NIH or NCATS. 


\title{
KANSAS JOURNAL of MEDICINE
}

\section{Charles Bengston, M.D.}

Assistant Professor

Department of Internal Medicine

University of Kansas Medical Center

Frontiers Postdoctoral Trainee Scholar (TLl)

\section{Evaluating the Adverse Effects of Hyperglycemia on the Airway Epithelium in Cystic Fibrosis and CF-Related Diabetes}

\author{
Received Feb. 7, 2020; Accepted for publication Feb. 10, 2020; Published online Feb. 26, 2020
}

\begin{abstract}
Introduction: Although causal linkage between hyperglycemia and decreased lung function in cystic fibrosis (CF) is unknown, it is thought to be related to excess inflammation and ion channel dysfunction.
\end{abstract}

Methods: We utilized complementary in vitro and in vivo approaches to assess the effects of hyperglycemia on the CF airway epithelium. In vitro: Primary CF bronchial epithelial (CFBE) cells at the air-liquid interface under hyperglycemic (HG; $255 \mathrm{mg} / \mathrm{dL}$ ) or normoglycemic $(\mathrm{NG} ; 100 \mathrm{mg} / \mathrm{dL}$ ) conditions. Airway surface liquid (ASL) was measured by meniscus scanning and apical K+ (BK) ion channel current was measured in Ussing chambers. In vivo: In CF subjects with or without CF related diabetes (CFRD), glycemic control was measured using continuous glucose monitoring.

Results: Nasal epithelial cells were collected and analyzed by qPCR for the expression of RAGE, TGF-1, and LRRC26, the regulatory subunit of BK. Compared to normoglycemia, CFBE cells under hyperglycemia show significantly reduced ASL (68.4 $\pm 17.4 \mu \mathrm{L}, \mathrm{NG}$ vs. $51.5 \pm 18.1 \mu \mathrm{L}$, HG; $<<0.05)$, a significant decrease in BK activity (-10.6 $\pm 0.8 \mu \mathrm{A} / \mathrm{cm} 2$, NG vs. $-1.6 \pm 0.5 \mu \mathrm{A} / \mathrm{cm} 2, \mathrm{HG})$, and a $69 \%$ increase in mRNA expression of RAGE ( $<0.05)$. From our in vivo studies, CF patients with and without CFRD showed significant negative correlation between the mRNA expressions of TGF-1 and LRRC26 (R2=0.73, $<<0.05)$.

Conclusions: In vitro data suggests hyperglycemia adversely affects airway hydration, in part, by impairing BK channel function. Reduction of LRRC26 expression in subjects with elevated TGF-1 and those with glycemic abnormalities point to BK as a target for therapeutic intervention in CFRD.

Funding Support: This work was supported by a CTSA grant from NCATS awarded to the University of Kansas for Frontiers: University of Kansas Clinical and Translational Science Institute (\#UL1TR002366). The contents are solely the responsibility of the authors and do not necessarily represent the official views of the NIH or NCATS. 
Clark Bloomer, B.S.

Project Manager - Genomics Core

Kansas Intellectual Developmental Disability Research Center

University of Kansas Medical Center

Frontiers Infrastructure Award Recipient

\section{University of Kansas Medical Center Genomics Core}

Received Feb. 7, 2020; Accepted for publication Feb. 10, 2020; Published online Feb. 26, 2020

\section{ABSTRACT}

The University of Kansas Medical Center - Genomics Core leveraged the Frontiers: KU Clinical and Translational Science Institute infrastructure award to purchase an Eppendorf MasterCycler Pro thermal cycler and Countess II Automated Cell Counter to support the single cell initiative of the Genomics Core at KUMC. The MasterCycler Pro and Countess II are used to support the 10X Genomics Chromium Controller for performing the full suite of 10X Genomics single cell applications which include Single Cell 3' Gene Expression, Single Cell 5 ’ Expression and V(D)J Enrichment and Single Cell ATAC - Chromatin Profiling.

The Countess II Automated Cell Counter is used to ensure that adequate cell counts and high viability of submitted samples are present. The Countess also allows visualization of any cellular debris or cell clumping which will interfere with a successful 10X emulsification procedure required for a successful single cell library preparation. The MasterCycler Pro has been sequestered to exclusively support single cell library preparations. The MasterCycler performs the initial single strand synthesis which incorporates the 10X GEM barcode and Unique Molecular Identifier (UMI), second strand synthesis and Illumina adapter ligation to complete the 10X library preparation.

These instruments have proven critical to the successful execution of 16 single cell projects in support of seven different investigators. Frontiers' investment in the single cell initiative has had a positive impact on cutting edge single cell expression and epigenetic research performed at the University of Kansas Medical Center.

Funding Support: This work was supported by a CTSA grant from NCATS awarded to the University of Kansas for Frontiers: University of Kansas Clinical and Translational Science Institute (\#UL1TR002366). The contents are solely the responsibility of the authors and do not necessarily represent the official views of the NIH or NCATS. 


\title{
KANSAS JOURNAL of MEDICINE
}

\author{
Prabhakar Chalise, Ph.D. \\ Assistant Professor \\ University of Kansas Medical Center \\ Department of Biostatistics and Data Science
}

Biostatistics, Epidemiology and Research Design (BERD) Trailblazer Award Recipient

Analysis of Mitochondrial Haplogroups with Alzheimer Disease Risk

Palash Sharma, M.S. ${ }^{1}$, Jonathan Mahnken, Ph.D. ${ }^{1}$, Dongwei Hui, Ph.D. ${ }^{2}$, Mary Michaelis, Ph.D. ${ }^{2}$, Elias Michaelis, M.D., Ph.D. ${ }^{2}$, Russell Swerdlow, M.D. ${ }^{3}$, Prabhakar Chalise, Ph.D. ${ }^{1}$

${ }^{1}$ University of Kansas Medical Center, Department of Biostatistics and Data Science, Kansas City, KS

${ }^{2}$ University of Kansas, Higuchi Biosciences Center, Lawrence, KS

${ }^{3}$ University of Kansas Medical Center, Department of Neurology, Kansas City, KS

Received Feb. 7, 2020; Accepted for publication Feb. 10, 2020; Published online Feb. 26, 2020

\begin{abstract}
Introduction: Alzheimer's disease (AD) is the most common form of irreversible, progressive dementia that affects memory loss, cognitive capabilities, and behavior. Although, significant progress has been made to understand genetic and clinical risk factors associated with AD, our understanding of AD remains incomplete resulting in ineffective prevention or cure.
\end{abstract}

Methods: Several studies have indicated that the polymorphisms in the mitochondrial DNA (mtDNA) play an important role in the mechanism of AD. Therefore, in order to understand the association of the mitochondrial genetic alteration with AD, we carried out cross-sectional association analysis using the clinical and mitochondrial single nucleotide polymorphism (SNP) data. We used the data collected at University of Kansas Alzheimer's Disease Center (KU ADC) to carry out the analyses. We also used the data from Alzheimer's Disease Neuroimaging Initiative (ADNI) for the replication and validation analyses.

Results: The KU ADC data consists of 146 AD and 265 cognitively normal subjects, and ADNI data has 244 AD and 242 controls. For both datasets, the haplogroups information for each subject and APOE $\varepsilon 4$ carrier status were also available.

Conclusions: We found that the rates of incidence of AD were statistically significantly different among the haplogroups, with differing strengths of association for different haplogroups. APOE $\varepsilon 4$ was significantly associated with AD overall ( $\mathrm{p}$-value $=1.9 \times 10-11)$ and the association varied by haplogroups (i.e., interaction). The results were consistent with the ADNI data. Our study has generated hypothesis and the data for further studies ahead to understand the etiology of AD.

Funding Support: This work was supported by a CTSA grant from NCATS awarded to the University of Kansas for Frontiers: University of Kansas Clinical and Translational Science Institute (\#ULITR002366). The contents are solely the responsibility of the authors and do not necessarily represent the official views of the NIH or NCATS. 
Sarah Dallas, Ph.D.

Lee M. and William Lefkowitz Endowed Professor

Department of Oral and Craniofacial Sciences,

University of Missouri-Kansas City School of Dentistry

Frontiers Infrastructure Award Recipient

\title{
Live Cell and Intravital Imaging Reveals Differences in Mitochondrial Redox State, Morphology and Number between Osteo- blasts and Osteocytes
}

Received Feb. 7, 2020; Accepted for publication Feb. 10, 2020; Published online Feb. 26, 2020

\begin{abstract}
Introduction: Age-related bone loss and associated fracture risk is an increasing concern worldwide. Oxidative stress causes mitochondrial damage and is associated with age-related degeneration in many cell systems, including bone cells.
\end{abstract}

Methods: To understand mitochondrial function in bone cells in situ, live-cell and intravital imaging of mitochondrial NADH and mitochondrial morphology was performed using transgenic mice expressing GFP in osteocytes as well as in MLO-Y4 osteocyte-like cells and OmGFP66 cells, which model osteoblast-to-osteocyte transition. NADH excitation and emission spectra in live osteocytes and osteoblasts matched well with pure NADH, with maximal multiphoton excitation at 740 - 760nm, resulting in blue NADH autofluorescence. With $760 \mathrm{~nm}$ excitation, maximal NADH emission occurred at 460nm. Metabolic challenge was performed with carbonylcyanide-3-chlorophenylhydrazone and sodium cyanide, which convert NADH to its maximally oxidized or reduced states.

Results: This showed that osteoblasts had a lower resting redox state, with $40 \%$ of their NADH in a reduced state, compared to osteocytes with $75-80 \%$. Live imaging using mitotracker Red-CMXRos showed that osteocytes have fewer mitochondria, lower mitochondrial area and $\%$ area, and increased solidity.

Conclusions: These data show that osteocytes in vivo under normoxic conditions have significant levels of mitochondrial NADH and show differences in mitochondrial number, morphology and function compared to osteoblasts. These live-cell imaging techniques can be used to determine changes in mitochondrial function during aging/cellular senescence and disease. These studies were supported by Frontiers equipment funding, including a Tokai-Hit CO2 incubation system, Leica 16x and 20x objectives and Imaris9.2 software which are available to other investigators through the UMKC Confocal Imaging Core.

Funding Support: This work was supported by a CTSA grant from NCATS awarded to the University of Kansas for Frontiers: University of Kansas Clinical and Translational Science Institute (\#ULITR002366). The contents are solely the responsibility of the authors and do not necessarily represent the official views of the NIH or NCATS. 


\title{
KANSAS JOURNAL of MEDICINE
}

\author{
Prasad Dandawate, Ph.D. \\ Research Assistant Professor \\ Department of Cancer Biology \\ University of Kansas Medical Center \\ Frontiers Infrastructure Award Recipient
}

Targeting Prolactin Receptor Using FDA-Approved Drugs: A Molecular Docking Approach and Drug Binding Studies

Received Feb. 7, 2020; Accepted for publication Feb. 10, 2020; Published online Feb. 26, 2020

\begin{abstract}
Introduction: Pancreatic cancer (PDAC) is the fourth leading cause of cancer-related deaths in the U.S. Gemcitabine remains the drug of choice for metastatic disease, but only little improvement is seen in survival rates. Hence, there is a dire need for novel therapeutics for PDAC. We found that PRLR is overexpressed in PDAC tissues and cell lines and knockdown of the receptor resulted in reduced tumor formation in mice. Hence, PRLR is a novel target for therapeutic interventions in PDAC.
\end{abstract}

Methods: As there is no small molecular inhibitor available for targeting the receptor, we developed a homology model of the intracellular domain of PRLR that encodes the JAK2 binding site, and then performed a virtual screening of small molecules using IDOCK and I-TASSER servers. Using Fragment-based drug design, we derived commercially available compounds for virtual screening. We selected several compounds that came out of the virtual screening and performed in vitro proliferation assays in the PDAC cells. We identified antipsychotic drugs including penfluridol that can inhibit the proliferation of PDAC cell lines. Moreover, pretreatment of these antipsychotic drugs inhibited PRL-induced STAT3 and ERK phosphorylation in PDAC cell lines. Further, we used cellular thermal shift assay and drug affinity binding assay to confirm the binding of Penfluridol and PRLR. We also used cell-free systems such as surface plasmon resonance (SPR) and magnetic relaxometry to confirm the drug binding. Moreover, Penfluridol treatment significantly decreased pancreatic tumor burden in animal models. In conclusion, we identified penfluridol is a potential drug candidate for targeting PRLR in PDAC.

Funding Support: This work was supported by a CTSA grant from NCATS awarded to the University of Kansas for Frontiers: University of Kansas Clinical and Translational Science Institute (\#ULITR002366). The contents are solely the responsibility of the authors and do not necessarily represent the official views of the NIH or NCATS. 
Kelsie Forbush, Ph.D.

Associate Professor

Department of Psychology

University of Kansas

Frontiers Dr. Lauren S. Aaronson Trailblazer Recipient
Victoria Perko ${ }^{1}$, Kelsie Forbush ${ }^{1}$, Sara Gould ${ }^{1,2}$, Brianne Richson ${ }^{1}$, Kylie Christian ${ }^{1}$, Danielle Chapa ${ }^{1}$, Laura Martin $^{3}$

${ }^{1}$ University of Kansas, Lawrence, KS

${ }^{2}$ Children’s Mercy Hospital, Kansas City, MO

${ }^{3}$ University of Kansas Medical Center, Kansas City, KS

Received Feb. 7, 2020; Accepted for publication Feb. 10, 2020; Published online Feb. 26, 2020

\begin{abstract}
Introduction: Anorexia nervosa (AN) is a serious condition associated with numerous medical complications, psychiatric comorbidity, and the highest mortality rate of any psychiatric disorder. Relapse rates remain high and for many, AN becomes an enduring condition. The only reliable prognostic indicator for AN is "rapid response" (i.e., weight gain over the first four weeks of treatment). Yet, there are no reliable predictors of rapid response at baseline. There is a pressing need to identify novel neuro-markers that confer early risk for poor treatment outcome.
\end{abstract}

Methods: Study goals were to systematically identify potential neuro-markers related to short-term AN outcomes by assessing brain activation associated with delayed discounting (capacity to delay immediate gratification), sensitivity to food reward (responses to rewarding foods and decisions to choose palatable foods), and threat sensitivity (heightened reactivity to potential danger or harm). The purpose of this study was to identify neural predictors of short-term treatment response in adolescents with AN. Participants were adolescent females with AN who entered their first course of eating-disorder treatment within the past six months.

Specific aims: 1) Test neural activation during tasks measuring delayed discounting, food reinforcement, and threat and 2) Identify prospective associations of task-performance and task-based neural activation with eating-disorder symptoms at one-month follow-up.

Conclusions: Differentiating neurobiological processes that contribute to AN chronicity may lead to the development of prognostic indicators at initial treatment presentation. These neuromarkers may improve clinical decision-making and allow providers to identify patients requiring more intensive treatment interventions to prevent chronicity and mortality.

Funding Support: This work was supported by a CTSA grant from NCATS awarded to the University of Kansas for Frontiers: University of Kansas Clinical and Translational Science Institute (\#ULITR002366). The contents are solely the responsibility of the authors and do not necessarily represent the official views of the NIH or NCATS. 


\title{
KANSAS JOURNAL of MEDICINE
}

\author{
Mark Johnson, Ph.D. \\ Professor and Chair \\ Department of Oral and Craniofacial Sciences, \\ University of Missouri-Kansas City School of Dentistry
}

Frontiers Infrastructure Award Recipient

\section{A High Throughput Assay for Quantitating $\beta$-catenin Signaling in the MLO-Y4 Osteocyte-like Cell Line}

Received Feb. 7, 2020; Accepted for publication Feb. 10, 2020; Published online Feb. 26, 2020

\begin{abstract}
Introduction: Osteocytes are the primary mechanosensory cell in bone and activate $\beta$-catenin signaling in response to mechanical loading of bone.

Methods: In order to understand the mechanism by which osteocytes activate $\beta$-catenin signaling, we have developed a stably transfected osteocyte cell line that expresses luciferase when $\beta$-catenin signaling occurs in the nucleus. To quantitate luciferase activity, we purchased a VICTOR Nivo Multimode Plate Reader (Perkin-Elmer) with the support of Frontiers funding. This instrument quantitatively measures luciferase activity (fluorescence and absorbance) in several plate formats, thus permitting high throughput screening of compounds that activate $\beta$-catenin signaling. We stably transfected the MLO-Y4 osteocyte-like cell line with the TOPflash vector pGL4.9[luc2P/TCF_LEFRE/Hygro] (Promega). Clone A3-F9 gave a dose response to increasing concentrations of Wnt3a (1-100ng/ml) and activation with 10mM LiCl gave the equivalent of a submaximal dose of Wnt3a (10ng/ml).
\end{abstract}

Results: We demonstrated that $\mathrm{C} 2 \mathrm{Cl} 2$ myotubes, but not myoblasts, produce a factor that activates $\beta$-catenin signaling two-fold using these TOPflash-MLO-Y4 cells, but in combination with Wnt3a (10 ng/ml; 10-fold activation) produces a 20-30-fold increase in activity measured in our luciferase assay. We demonstrated that production of this factor by $\mathrm{C} 2 \mathrm{C} 12$ myotubes is partially regulated by mTOR signaling. We are currently seeking to identify this factor using our TOPflash-MLO-Y4 cells.

Conclusions: The acquisition of the VICTOR Nivo Multiplate reader provides us the means to conduct a high throughput screening approach to identify this factor(s). This instrument is part of a Core in the UMKC School of Dentistry Department of Oral and Craniofacial Sciences and available to other investigators for their studies.

Funding Support: This work was supported by a CTSA grant from NCATS awarded to the University of Kansas for Frontiers: University of Kansas Clinical and Translational Science Institute (\#UL1TR002366). The contents are solely the responsibility of the authors and do not necessarily represent the official views of the NIH or NCATS. 
Eleanor Leavens, Ph.D.

Department of Population Health

University of Kansas Medical Center

Frontiers Postdoctoral Fellow (TL1)

Exploratory Evaluation of an Online Educational Intervention for JUUL Use

Matthew J. Carpenter, Ph.D. ${ }^{1}$, Tracy T. Smith, Ph.D. ${ }^{1}$, Nikki Nollen, Ph.D. ${ }^{2}$, Eleanor Leavens, Ph.D. ${ }^{2}$

${ }^{1}$ Medical University of South Carolina, Charleston, SC

${ }^{2}$ University of Kansas Medical Center, Kansas City, KS

Received Feb. 7, 2020; Accepted for publication Feb. 10, 2020; Published online Feb. 26, 2020

\begin{abstract}
Introduction: Initiation of JUUL use by young adults is one of the most significant issues of concern within the debate on vaping. Despite the proliferation of products and the surge in prevalence, no studies have investigated individual-level interventions or prevention strategies for pod-mod use.
\end{abstract}

Methods: Participants ( $\mathrm{N}=947)$ were young adults (<30 years old) recruited from Amazon's Mechanical Turk based on smoking (never, former, and current smokers) and JUUL use status (never and current users). In a pre-post design, participants completed baseline assessments, were presented with a brief JUUL-specific educational intervention and completed post-assessment measures. Primary outcomes were changes in JUUL knowledge, perceived harmfulness, intentions for future use, and motivation to change.

Results: Participants (Mean age $=26.1)$ were male $(57 \%)$ and White $(75 \%)$. Overall, the intervention increased JUUL-related knowledge, risk perceptions, commitment to quitting, and readiness to quit JUUL (all $\mathrm{p} \leq 0.001$ ). Similarly, participants showed decreased interest in future JUUL use, interest in purchasing JUUL, and interest in future regular JUUL use (all $\mathrm{p} \leq 0.001$ ). There were significant Time X Group interactions for JUUL-related knowledge ( $<$ 0.001), with never JUUL/never smokers showing the greatest increase in product knowledge following the intervention. However, no other interaction effects were significant.

Discussion: The intervention was effective in increasing knowledge and risk perceptions while reducing intentions for future use. The intervention was most effective in increasing knowledge among non-users, suggesting that brief educational interventions may be useful tools for preventing pod-mod initiation while more intensive interventions may be necessary to alter attitudes and use behaviors among current users.

Funding Support: This work was supported by a CTSA grant from NCATS awarded to the University of Kansas for Frontiers: University of Kansas Clinical and Translational Science Institute (\#ULITR002366). The contents are solely the responsibility of the authors and do not necessarily represent the official views of the NIH or NCATS. 


\title{
KANSAS JOURNAL of MEDICINE
}

\author{
Charley Lewis, MPH \\ Research Instructor \\ Center for American Indian Community Health \\ University of Kansas Medical Center
}

Frontiers Clinical and Translational Pilot Research Grant Recipient

\section{American Indian College Students' Knowledge, Attitudes, Beliefs, and Behaviors Surrounding Alternative Tobacco Products}

Received Feb. 7, 2020; Accepted for publication Feb. 10, 2020; Published online Feb. 26, 2020

\begin{abstract}
Introduction: American Indians (AI) have the highest rates of smoking and smokeless tobacco (SLT) use among all major racial/ethnic groups. There is limited information about the use of alternative tobacco (AT) products such as electronic cigarettes. The objective of this pilot study is to understand the knowledge, attitudes, beliefs, and behaviors (KABB) surrounding AT product use among AI college students.

Methods: We conducted 19 focus groups with AI college students age 18 and older. Participants were stratified by AT use, cigarette use, and never users of tobacco products. Focus groups were audio-recorded and transcribed verbatim in preparation for coding and standard text analysis. Participants completed a short survey that collected information on demographics and KABB about tobacco products. Surveys were analyzed with frequencies and measures of central tendencies.
\end{abstract}

Results: Participants included 105 AI college students. Participant's mean age was 21 years and $50 \%$ were female. Focus groups ranged in size from two to nine. Related to e-cigarette use, participants cited flavors, discreteness, stress, and social aspects as factors for use. Survey results indicate that 35\% of participants were current e-cigarette users, 30\% were current smokers, and 9\% were current SLT users.

Conclusion: This pilot study confirms that AI college students are following a similar trend to other racial/ethnic groups in terms of AT use. It is important that we obtain estimates of AT use and include AI in prevention and cessation efforts. This study will help develop appropriate prevention and cessation messaging for this population.

Funding Support: This work was supported by a CTSA grant from NCATS awarded to the University of Kansas for Frontiers: University of Kansas Clinical and Translational Science Institute (\#UL1TR002366). The contents are solely the responsibility of the authors and do not necessarily represent the official views of the NIH or NCATS. 
Tara Lin, M.D., M.S.

Associate Professor

University of Kansas Medical Center

University of Kansas Cancer Center

Frontiers Clinical and Translational Pilot Research Grant Recipient

Low Dose Daunorubicin Targets the Leukemia Stem Cell in AML

Tara L. Lin, M.D., ${ }^{1,2}$,John M. Perry, Ph.D. ${ }^{4}$, Xi He, Ph.D. ${ }^{3}$, Gregory Reed, Ph.D. ${ }^{1}$, Na Zhang, Ph.D. ${ }^{1}$, Scott Weir, Pharm.D., ${ }^{1,5}$, Joseph P. McGuirk, D.O., ${ }^{1,2}$ Linheng Li, Ph.D., ${ }^{1,3}$

${ }^{1}$ University of Kansas Cancer Center, Kansas City, KS

${ }^{2}$ University of Kansas, Division of Hematologic Malignancies \& Cellular Therapeutics, Kansas City, KS

${ }^{3}$ Stowers Institute for Medical Research, Kansas City, MO

${ }^{4}$ Children’s Mercy Hospital and Clinics, Kansas City, MO

${ }^{5}$ University of Kansas, Division of Medical Oncology, Kansas City, KS

Received Feb. 7, 2020; Accepted for publication Feb. 10, 2020; Published online Feb. 26, 2020

\section{ABSTRACT}

Introduction: A proof of concept trial (NCTO2914977) was designed to measure the ability of low dose DNR to inhibit pS552- $\beta$-catenin in LSCs of adult relapsed/refractory AML patients. A protocol amendment added a second cohort of patients with newly diagnosed AML undergoing standard chemotherapy with DNR and cytarabine (7+3 induction).

Methods: Treatment in each cohort consisted of DNR given at a dose of $6.75 \mathrm{mg} / \mathrm{m} 2 /$ day x five days. Patients with relapsed/refractory AML had bone marrow aspiration pre-treatment prior to day 1, followed by DNR on days 1-5, and a post-treatment bone marrow aspiration on day 8. Patients with newly diagnosed AML were treated with conventional $7+3$ chemotherapy.

Results: A sample from the day 14 bone marrow was collected. DNR was given on days 15 - 19. Bone marrow aspiration was performed with blood count recovery and samples sent for LSC marker assessment.

Discussion: In this study, we will determine the safety, pharmacokinetics, and pharmacodynamic effects of low dose DNR. The LSC population and phosphorylation status of $\beta$-catenin will be measured pre-and post-treatment. Two or more prior induction attempts are required for study entry for refractory patients; relapsed patients require only one prior induction. The trial completed its accrual goal of 18 patients. Low-dose DNR treatment was well-tolerated with no unexpected adverse events. In this pilot proof of concept trial, we will demonstrate the feasibility of serial bone marrow aspiration to measure biomarkers of LSC response.

Funding Support: This work was supported by a CTSA grant from NCATS awarded to the University of Kansas for Frontiers: University of Kansas Clinical and Translational Science Institute (\#UL1TR002366). The contents are solely the responsibility of the authors and do not necessarily represent the official views of the NIH or NCATS. 


\title{
KANSAS JOURNAL of MEDICINE
}

\section{Crystal Lumpkins, Ph.D., M.A.}

Associate Professor

Center for American Indian Community Health

Department of Family Medicine \& Community Health

University of Kansas Medical Center

Frontiers Pilot and Collaborative Studies Funding Program Award Recipient

\section{In Our Sacred Voice: An Exploration of Tribal and Community Leader Perceptions as Health Communicators of Disease Prevention among American Indians in the Plains

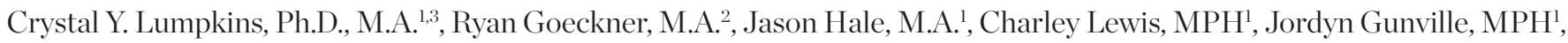 River Gunville, B.A. ${ }^{1}$, Chris M. Daley, Ph.D., M.A. ${ }^{1}$ \\ ${ }^{1}$ University of Kansas Medical Center, Center for American Indian Community Health, \\ Department of Family Medicine \& Community Health, Kansas City, KS \\ ${ }^{2}$ Ohio State University, Columbus, OH \\ ${ }^{3}$ University of Kansas, William Allen White School of Journalism and Mass Communication, Lawrence, KS}

Received Feb. 7, 2020; Accepted for publication Feb. 10, 2020; Published online Feb. 26, 2020

\begin{abstract}
Introduction: American Indians (AI) are disproportionately and significantly impacted by disease morbidity, mortality and poor behavioral health outcomes. Based on data from the National Vital Statistics Reports, the leading cause of death among AI is cancer (19\%), followed by heart disease (18\%). Regionally, health disparities among AIs reflect national statistics. Health communication targeted to address these health disparities exist; however, few evidence-based strategies integrate holistic approaches. The impact of health communication research and practice is evolving and continues to emerge as a core part of health promotion. Strategies that appeal and increase persuasiveness of health messages are more likely to penetrate existing cultural beliefs and positively influence health behavior outcomes. The objective of this study was to explore how tribal and elder leaders throughout the Plains viewed themselves as health communicators within their communities.
\end{abstract}

Methods: Members of the Center for American Indian Community Health conducted 39 in-depth interviews from December 2017 to December 2018 with members of federally recognized tribes living in reservation communities, as well as urban tribal communities across the region.

Results: Results from this sample show that these individuals do not see themselves as the "authority" health communicator within their tribe and community. The term health promoter was viewed as problematic in Native communities for cultural reasons.

Conclusion: Overarching themes included seeing themselves as communicators of holistic health, specific health prevention programs, access, targeted communication and health promoters as a collective unit. Collective social and cultural authority in tailored messages is perceived to bolster health communication and positively impact health outcome.

Funding Support: This work was supported by a CTSA grant from NCATS awarded to the University of Kansas for Frontiers: University of Kansas Clinical and Translational Science Institute (\#UL1TR002366). The contents are solely the responsibility of the authors and do not necessarily represent the official views of the NIH or NCATS. 
Crystal Lumpkins, Ph.D., M.A.

Associate Professor

Center for American Indian Community Health

Department of Family Medicine \& Community Health

University of Kansas Medical Center

Frontiers Pilot and Collaborative Studies Funding Program Award Recipient

\title{
Not About Us, Without Us - An Exploratory Study of Underserved and Minority Patients' Views of Cancer-Related Genetic Counseling and Testing Communication
}

Crystal Y. Lumpkins, Ph.D., M.A. ${ }^{1}$ Alisdair Philp, Ph.D., M.S., CGC ${ }^{2}$, Kim Kimminau, Ph.D. ${ }^{1}$, Mariana Ramirez--Mantilla, LMSW3,5, Reem Mustafa, M.B.B.S., Ph.D., MPH², Andrew Godwin, Ph.D. ${ }^{4}$, Yani Vazquez, MBA7, Nancy Washington ${ }^{8}$, Kim Jones ${ }^{6,8}$, Shaton Freeman ${ }^{6,8}$ University of Kansas Medical Center, Kansas City, KS

${ }^{1}$ Department of Family Medicine and Community Health

${ }^{2}$ Department of Internal Medicine

${ }^{3}$ Department of Population Health

${ }^{4}$ Department of Pathology and Laboratory Medicine

${ }^{5}$ Juntos Center for Advancing Latino Health, Kansas City, KS

${ }^{6}$ Sisters Living Beyond the Ribbon, Kansas City, MO

${ }^{7}$ Hispanic Chamber of Commerce of Greater Kansas City, Kansas City, MO

${ }^{8}$ University of Kansas Cancer Center, Patient and Investigator Voices Organizing Together (PIVO'T) Team, Kansas City, KS

Received Feb. 7, 2020; Accepted for publication Feb. 10, 2020; Published online Feb. 26, 2020

\begin{abstract}
Introduction: Minority and underserved populations carry the largest cancer burden for the United States (ACS, 2016). Genetic counseling, testing and technologies show promise to help reduce cancer through personalized medicine opportunities however disproportions of genetic services, uptake and research about genetic testing among these populations exist (Ricker, et al., 2018). Inequitable cancer-related genetic and genomic testing (CGT) communication among minority and underserved populations and other barriers (e.g., genetic counselor bias) may contribute to CGT disparities. Exploring existing attitudes, perceptions and beliefs toward CGT among minority and underserved patient populations may bolster strategies to address communication inequities. The study aims were to explore an under-researched area of precision medicine, i.e., the communication of genetic risk factors among minority and underserved patient populations with high to moderate risk and disseminate and share findings with the community.
\end{abstract}

Methods: A Community Advisory Board comprised of researchers, cancer survivors and family members of cancer patients met from December 2018 - March 2019 to discuss research design and data collection. Six stratified focus group discussions (N = 53) were held March - May 2019 where a pre-focus group survey was administered.

Results: Survey results show most had not talked to their doctor/health care provider about genetic testing (87\%). African Americans (AA) and Latinos expressed a lack of awareness and knowledge about CGT and the importance of cultural tailoring.

Conclusion: Separate themes that emerged among Latinos included immigration status and language as barriers to CGT. Among AA, participants were highly distrustful of medical personnel and expressed outrage about the lack of recommendation for CGT.

Funding Support: This work was supported by a CTSA grant from NCATS awarded to the University of Kansas for Frontiers: University of Kansas Clinical and Translational Science Institute (\#UL1TR002366). The contents are solely the responsibility of the authors and do not necessarily represent the official views of the NIH or NCATS. 


\title{
KANSAS JOURNAL of MEDICINE
}

\section{Laura Martin, Ph.D.}

Associate Professor

Department of Population Health

University of Kansas Medical Center

Frontiers Clinical and Translational Pilot Research Grant Recipient

\section{Changing how the Brain Responds When Making Decisions: Translating Neuroscience to Population Health}

Laura E. Martin, Ph.D. ${ }^{1}$, Morgan G. Brucks, B.A.', Andrew Fox, Ph.D. ${ }^{1}$, Vlad B. Papa, B.A.', Noreen D. Mdege, Ph.D., MPH², Austin S. Baldwin, Ph.D., Elisa M. Trucco, Ph.D. ${ }^{3}$, Nina A. Cooperman, Psy.D. ${ }^{5}$, Angelos P. Kassianos, Ph.D. ${ }^{6}$, Sara M. Levens, Ph.D.

${ }^{1}$ University of Kansas Medical Center, Kansas City, KS

${ }^{2}$ University of York, United Kingdom

${ }^{3}$ Southern Methodist University, Dallas, TX

${ }^{4}$ Florida International University, Miami, FL

${ }^{5}$ Rutgers University, New Brunswick, NJ

${ }^{6}$ University College London, United Kingdom

${ }^{7}$ University of North Carolina-Charlotte, Charlotte, NC

Received Feb. 7, 2020; Accepted for publication Feb. 10, 2020; Published online Feb. 26, 2020

\begin{abstract}
Introduction: Engaging in healthy behaviors is not always rewarding (e.g., carrot vs. cake). Neural models of healthy behaviors focus on the balance between reward and regulation brain regions. This pilot study examines the engagement of reward and regulation regions during the evaluation of food and nonfood cues before and after a guided imagery exercise targeting positive associations with food and regulation. Our long-term goal is to understand the interaction between positive associations with healthy foods and thinking about future rewards may influence healthy decision-making. The objective of the current study is to empirically test the combined effects of positive affect and positive episodic future thinking on brain activation.
\end{abstract}

Methods: The project examined the effect of positive affect and episodic future thinking guided imagery on brain activation in regulation and reward regions. Participants included individuals with a body mass index greater than $25(\mathrm{n}=20)$.

Results: Functional neuroimaging showed reduced activation in regulation-related brain regions (i.e., dorsomedial prefrontal cortex) to healthy and unhealthy food following guided imagery.

Conclusions: These results suggest more efficient regulation-related brain activation after positive episodic future thinking guided imagery.

Funding Support: This work was supported by a CTSA grant from NCATS awarded to the University of Kansas for Frontiers: University of Kansas Clinical and Translational Science Institute (\#ULITR002366). The contents are solely the responsibility of the authors and do not necessarily represent the official views of the NIH or NCATS. 
Reem Mustafa, M.D., MPH, Ph.D.

Associate Professor

Department of Internal Medicine

Kidney Institute

University of Kansas Medical Center

Frontiers Dr. Lauren S. Aaronson Trailblazer Recipient

\title{
Engaging Patients and Defining Outcomes: A Minority Engagement Effort
}

Received Feb. 7, 2020; Accepted for publication Feb. 10, 2020; Published online Feb. 26, 2020

\begin{abstract}
Introduction: The Establishing Meaningful Patient-centered Outcomes With Relevance for patients with Polycystic Kidney Disease (EMPOWER PKD) initiative aims to engage PKD stakeholders and patients to learn about health priorities, insurance issues, and patient engagement.

Methods: We utilized semi-structured focus groups. We developed and pre-piloted a guide that allowed for both conversational flow and consistency in questions among groups. We audio-recorded each group and transcribed the conversations verbatim. We performed an inductive thematic analysis. Two investigators completed all data coding independently and in duplicate. We compare the results of an ethnically diverse focus group to the other seven that mainly included Caucasian participants.
\end{abstract}

Results: 14 individuals participated in this part of the study. Of these, (64.2\%) reported having PKD, and (14.2\%) being caregivers. The mean age of participants was 48.8 (range 29 - 75) years. The group included 85.75\% African American and 7.1\% reported more than one race.

Conclusions: It was challenging to recruit this group and it required establishing a relation with community nephrologists and faith-based organizations leads. Multiple participants stated that they would not have participated if the group was not exclusively ethnically diverse as they fear that their opinion may be outnumbered by a Caucasian majority. Some discussed believing that PKD was a "white" disease. Many discussed hiding their diagnosis which affected their activation and engagement and came with unanticipated psychological impacts. Participants unanimously described lack of trust-worthy, easy to understand educational resources for minorities as a main barrier to engagement.

Funding Support: This work was supported by a CTSA grant from NCATS awarded to the University of Kansas for Frontiers: University of Kansas Clinical and Translational Science Institute (\#ULITR002366). The contents are solely the responsibility of the authors and do not necessarily represent the official views of the NIH or NCATS. 


\title{
KANSAS JOURNAL of MEDICINE
}

\author{
Ebony Onianwa, B.S. \\ Department of Population Health \\ University of Kansas Medical Center \\ Frontiers Predoctoral Trainee Scholar (TLl)
}

\section{Race-Related Stress and Food Decision Making in African American Women}

Received Feb. 7, 2020; Accepted for publication Feb. 10, 2020; Published online Feb. 26, 2020

\begin{abstract}
Introduction: The highest rates of obesity in the U.S. are among black women with a prevalence of $54.8 \%$. Psychosocial factors, such as stress from racism, have been proposed as contributors to this disparity, but the mechanisms are unclear. The objective of this study is to understand the relationship between race-related stress and food choice among black women.
\end{abstract}

Methods: Forty-five participants were asked to come in fasted for two appointments, randomized to the order of stress exposure in a within subject design. Forty images for each condition were collected from two validated picture sets (International Affective Picture set and Socio Moral Image Database). The images were shown in random order and contained either racially charged scenes (e.g., Ku Klux Klan) or nonracially charged scenes (e.g., aggressive dog) matched on valence (pleasant vs. unpleasant) and arousal (physiologically stimulating). Food choice and food demand tasks were completed pre and post stress exposure. Participants were surveyed on their stress levels, past experiences of racism, and trauma.

Anticipated Results: It is expected that preferences for unhealthy foods will be greater after race related stress exposure compared to the other stress condition. We anticipate that those who have higher BMI's are more likely to be affected in their changes in eating behaviors in both stress exposure states, with a larger impact from the race related stress induction. Data collection is ongoing.

Funding Support: This work was supported by a CTSA grant from NCATS awarded to the University of Kansas for Frontiers: University of Kansas Clinical and Translational Science Institute (\#ULITR002366). The contents are solely the responsibility of the authors and do not necessarily represent the official views of the NIH or NCATS. 


\section{Shariska Petersen, M.D.}

Department of Obstetrics and Gynecology

University of Kansas Medical Center

Frontiers Postdoctoral Fellow (TL1)

\section{Low Socioeconomic Status and Residential Distance of Less Than 10 Miles From a Frontier-State NCI-Designated Cancer Center is Associated with Worse Ovarian Cancer Survival}

Received Feb. 7, 2020; Accepted for publication Feb. 10, 2020; Published online Feb. 26, 2020

\section{ABSTRACT}

Introdution: To examine the impact of residential distance and socioeconomic status on survival outcomes for patients receiving treatment for ovarian cancer at an NCI-CC.

Methods: Patients who were treated for ovarian cancer at a single institution from 2010 - 2015 were identified. Age at diagnosis, insurance status, and distance from the patient's home to the institution were abstracted. Clinical data including stage at diagnosis, surgical status, chemotherapy cycles, dates of diagnosis, recurrence, and death were obtained. Patients treated at other institutions and those with nonepithelial pathology were excluded. Patients were stratified into three groups based on distance from the institution. Overall survival (OS) and progression free survival (PFS) were generated by Kaplan Meier survival curves and Cox proportional hazard models using SAS v9.4.

Results: Two hundred and three patients were identified. Survival analysis based on distance demonstrated that patients who lived less than 10 miles from the institution have worse survival $(\mathrm{p}=0.0412)$. Lower median income $(<56,179)$ regardless of distance to institution was also associated with worse survival, $\mathrm{p}=0.0283$. After adjusting for stage of disease, age at diagnosis, surgery status, chemotherapy resistance, income quartile and presence of comorbid conditions, distance $<10$ miles from the institution was still a significant predictor of worse overall survival, HR $2.6295 \%$ CI (1.333 - 5.142), p = 0.0052.

Conclusion: Among patients who received guideline-adherent care for ovarian cancer, lower median income and residential distance less than 10 miles to an NCI-CC was associated with worse overall survival.

Funding Support: This work was supported by a CTSA grant from NCATS awarded to the University of Kansas for Frontiers: University of Kansas Clinical and Translational Science Institute (\#ULITR002366). The contents are solely the responsibility of the authors and do not necessarily represent the official views of the NIH or NCATS. 


\title{
KANSAS JOURNAL of MEDICINE
}

\section{Timothy Pleskac, Ph.D.}

Professor

Department of Psychology

University of Kansas

Frontiers Infrastructure Award Recipient

\author{
Understanding Race Bias in the Decision to Shoot with an Integrated Model of Decision Making \\ Timothy J. Pleskac, Ph.D. ${ }^{1}$, Sergej Grunevski, B.S. ${ }^{1}$, Taosheng Liu, Ph.D. ${ }^{2}$, Joseph Cesario, Ph.D. ${ }^{2}$ \\ 'University of Kansas, Lawrence, KS \\ ${ }^{2}$ Michigan State University, East Lansing, MI \\ Received Feb. 7, 2020; Accepted for publication Feb. 10, 2020; Published online Feb. 26, 2020
}

\section{ABSTRACT}

Introduction: The shooting of unarmed citizens by police officers is a topic at the forefront of public awareness. The disproportionate rate at which unarmed Black citizens are shot is believed to reflect widespread racial bias on behalf of officers, which erodes public trust and reduces policing effectiveness.

Methods: We have developed an Attention-integrated Model-based Shooting Simulator (AiMSS) to gain a deeper understanding of the mechanisms underlying the decision to shoot. The AiMSS combines computational models of decision making, visual psychophysics and eye-tracking methods, and an immersive decision simulator to map the processes police officers use to decide to shoot.

Results: Results with the AiMSS reveal that policing scenario and suspect behavior accounted for the most variation in decisions. Process level measures show little evidence of an initial bias to shoot Black suspects. Instead, results reveal a diminished ability to distinguish objects held by Black suspects.

Conclusions: This work emphasizes the importance of contextual factors in the decision to shoot and highlights how past experimental studies on racial bias have neglected critical inputs into officer deadly force decisions.

Funding Support: This work was supported by a CTSA grant from NCATS awarded to the University of Kansas for Frontiers: University of Kansas Clinical and Translational Science Institute (\#ULlTR002366). The contents are solely the responsibility of the authors and do not necessarily represent the official views of the NIH or NCATS. 


\section{Eduardo Rosa-Molinar, Ph.D.}

Professor and Director

Emerging Imaging Technologies and Applications Laboratory and Microscopy and Analytical Imaging Research Resource Core

Laboratory

University of Kansas

Frontiers Infrastructure Award Recipient

\title{
A Developing Shared Resource: Emerging Imaging Technologies and Applications Laboratory
}

Received Feb. 7, 2020; Accepted for publication Feb. 10, 2020; Published online Feb. 26, 2020

\begin{abstract}
The Emerging Imaging Technologies and Applications Laboratory (EITAL) is a shared resource serving as a proof-of-concept hub for performing innovative and rigorous fundamental cancer biology research at subcellular to cellular length-scales in conjunction with the development and translation of advanced imaging technologies and applications. The scientific staff of EITAL are experts in developing and improving reagents, methods, and optical and electron microscopic imaging technologies, antibody development, screening, and characterization, immunohistochemistry, immunocytochemistry, correlative optical and electron microscopy, immunoelectron microscopy, workflow development, and experimental design and data analysis of imaging studies. EITAL promotes synergies between its imaging technology developers and University of Kansas Cancer Center (KUCC) cancer biology researchers. EITAL also provides research training and career development programs to ensure a talented and diverse workforce engaged in cellular tumor cancer biology imaging research. The collaboration between EITAL and the University of Kansas (KU) Microscopy and Analytical Imaging Research Resource Core Laboratory (MAI) imaging scientists, enables imaging research at the level of the whole animal, individual cells, as well as molecules inside the cells, and provides instrumentation and expertise for optical and electron microscopies, resulting in multi-scale/multi-dimensional correlative imaging research of living and fixed cells and tissues. The KUCC Support Grant (P30 CAl68524) supports EITAL.
\end{abstract}

Funding Support: This work was supported by a CTSA grant from NCATS awarded to the University of Kansas for Frontiers: University of Kansas Clinical and Translational Science Institute (\#ULITR002366). The contents are solely the responsibility of the authors and do not necessarily represent the official views of the NIH or NCATS. 


\title{
KANSAS JOURNAL of MEDICINE
}

\section{Robin Shafer, Ph.D.}

Schiefelbusch Institute for Life Span Studies

University of Kansas

Frontiers Postdoctoral Fellow (TL1)

\author{
Influence of Vision and Proprioception on Motor Control in ASD \\ Robin L. Shafer, Ph.D., , Zheng Wang, Ph.D. ${ }^{3}$, Matthew W. Mosconi, Ph.D. ${ }^{1,2,4}$ \\ ${ }^{1}$ Schiefelbusch Institute for Life Span Studies, University of Kansas, Lawrence, KS \\ ${ }^{2}$ Kansas Center for Autism Research and Training, University of Kansas, Lawrence, KS \\ ${ }^{3}$ Department of Occupational Therapy, University of Florida, Gainesville, FL \\ ${ }^{4}$ Clinical Child Psychology Program, University of Kansas, Lawrence, KS \\ Received Feb. 7, 2020; Accepted for publication Feb. 10, 2020; Published online Feb. 26, 2020
}

\section{ABSTRACT}

Introduction: Sensorimotor integration deficits are common in Autism Spectrum Disorders (ASD). Evidence exists for an over-reliance on both visual and proprioceptive feedback during motor control in ASD, suggesting deficits in the modulation of sensory feedback processing and inability to use the most reliable input. This study aims to test this hypothesis.

Methods: Forty persons with ASD (10 - 33 yrs) and 25 age-, sex- and nonverbal IQ-matched controls completed precision gripping tasks. They squeezed a force sensor with their index finger and thumb and tried to match their force output to a target force. Visual feedback was presented on a computer screen across low, medium, and high gain levels; the force output bar moved a greater distance per change in force at higher gains. A stationary bar represented the target force. $80 \mathrm{~Hz}$ tendon vibration was applied at the wrist to create a proprioceptive illusion of muscle contraction. Force regularity (approximate entropy; ApEn) was examined.

Results: Preliminary results from 18 participants with ASD and 13 controls indicate a Group x Tendon Vibration x Visual Gain interaction for ApEn ( $\mathrm{F}=1.559, \mathrm{p}=0.023)$. Controls showed increased ApEn during 80Hz tendon vibration at low visual gain but decreased ApEn with vibration at high visual gain. Individuals with ASD show slight increases in ApEn with 80Hz vibration across visual conditions.

Conclusions: Early findings indicate that controls shift to a secondary source of sensory feedback (e.g., proprioception) when the primary source (e.g., vision) is degraded. Persons with ASD fail to dynamically recalibrate feedback processes across sensory systems when feedback conditions change.

Funding Support: This work was supported by a CTSA grant from NCATS awarded to the University of Kansas for Frontiers: University of Kansas Clinical and Translational Science Institute (\#UL1TR002366). The contents are solely the responsibility of the authors and do not necessarily represent the official views of the NIH or NCATS. 
Assistant Professor

Department of Population Health

University of Kansas School of Medicine-Wichita

Diversity Trailblazer Award Recipient

\author{
Health through Enhancing Awareness and Learning about Breast and Cervical Cancer Screening among \\ African American Women (HEAL) \\ Sharla Smith, MPH, Ph.D. ${ }^{1}$, Jannette Berkley-Patton, Ph.D. ${ }^{2}$, Megha Ramaswamy, MPH, Ph.D. ${ }^{3}$, Joi Wickliffe, MPH ${ }^{3}$ \\ ${ }^{1}$ University of Kansas School of Medicine-Wichita, Wichita, KS \\ ${ }^{2}$ University of Missouri-Kansas City, Kansas City, MO \\ ${ }^{3}$ University of Kansas School of Medicine, Kansas City, KS
}

Received Feb. 7, 2020; Accepted for publication Feb. 10, 2020; Published online Feb. 26, 2020

\begin{abstract}
Introduction: African American women experience higher incidence and mortality rates of cervical and breast cancer. The purpose of this study is to develop and implement a breast and cervical cancer screening intervention to increase awareness, uptake of screening, and followup among an African American female church-population in Sedgwick County, Kansas.
\end{abstract}

Methods: Academic researchers recruited local pastors $(\mathrm{N}=11)$ to develop a faith taskforce. Using intervention mapping, we conducted three focus groups with the taskforce to assess barriers and facilitators to seeking breast and cervical screenings and multi-component strategies to improve education and awareness of breast and cervical cancer screening recommendations, screening rates, and follow-up among African American women.

Preliminary Results: Of the 11 faith taskforce pastors, three were females. The key identified themes focused on barriers and facilitators to implementation of church-based cervical/breast cancer interventions, such as time constraints, limited expertise in conducting faith-based interventions, and lack of awareness on burden of cancer in the community, awareness of screening recommendations, and support/accountability in cancer screening and follow-up. Participants also indicated that a mobile phone app to track screenings, follow-up appointments, and promote education about risk factors and a church health navigator to assist with app utilization and track screenings and follow-up appointments would need to be primary intervention components.

Conclusions: Findings from the taskforce focus groups highlight the need for multi-component church-based interventions that include a mHealth app and church health navigators to improve awareness and adherence to breast/cervical cancer screening recommendations and follow-up among African American female church populations.

Funding Support: This work was supported by a CTSA grant from NCATS awarded to the University of Kansas for Frontiers: University of Kansas Clinical and Translational Science Institute (\#UL1TR002366). The contents are solely the responsibility of the authors and do not necessarily represent the official views of the NIH or NCATS. 


\title{
KANSAS JOURNAL of MEDICINE
}

\author{
Jason Springer, M.D., M.S. \\ Assistant Professor \\ Division of Allergy, Clinical Immunology and Rheumatology \\ Department of Internal Medicine \\ University of Kansas Medical Center
}

Frontiers Clinical and Translational Pilot Research Grant Recipient

\author{
RITUXimab IMmunogenicity in ANCA-associated Vasculitis (RITUXIMAV) \\ Jason Springer, M.D., M.S., Ryan Funk, Pharm.D., Ph.D. \\ Received Feb. 7, 2020; Accepted for publication Feb. 10, 2020; Published online Feb. 26, 2020
}

\begin{abstract}
Introduction: Rituximab (RTX) is an effective maintenance therapy in forms of ANCA-associated vasculitis (AAV), specifically granulomatosis with polyangiitis (GPA) and microscopic polyangiitis (MPA) (MAINRITSAN trial). Sixteen percent of patients will relapse despite RTX. Infliximab, an anti-chimeric antibody like RTX, is associated with a high rate of anti-drug antibody formation which effects its efficacy. The aims of this study are to a) evaluate the incidence of immunogenicity toward rituximab in AVV; and b) evaluate the relationship between anti-RTX antibodies and drug response. Herein we present the baseline data.
\end{abstract}

Methods: Inclusion criteria: a) meet either 1990 ACR and/or 2012 revised CHCC criteria for GPA or MPA; b) remission at entry; c) received Rituximab in last 12 months; d) plan to continue Rituximab; and e) no contraindications for receiving further RTX infusions.

Results: 30 participants (25 GPA and 5 MPA) were enrolled. ANCA by EIA was MPO positive in 14 (47\%), PR3 positive in 13 (43\%) and negative in 3 (10\%). Mean age at enrollment was 62.5 years (IQR 46.5 - 67.5). 70\% were female. The average immunoglobulin G (IgG) at baseline was $663 \mathrm{mg} / \mathrm{dl}$ (95\% CI 566 - 760). 67\% of participants had undetectable B-cells at baseline. Average time between the last Rituximab infusion and the first study Rituximab infusion was 34 weeks (95\% CI 28 - 40 weeks).

Conclusions: At study baseline most of the participants were found to be B-cell depleted and mildly hypogammaglobulinemic. The average duration since the last Rituximab infusion at entry (8.5 months) was slightly longer than in the MAINRITSAN trial (six months).

Funding Support: This work was supported by a CTSA grant from NCATS awarded to the University of Kansas for Frontiers: University of Kansas Clinical and Translational Science Institute (\#ULITR002366). The contents are solely the responsibility of the authors and do not necessarily represent the official views of the NIH or NCATS. 
Jeffrey Statland, M.D.

Assistant Professor

Department of Neurology

University of Kansas Medical Center

Institute for Advancing Medical Innovation (IAMI) Trailblazer Award Recipient

\section{Open-Label Pilot Study of Ranolazine for Cramps in Amyotrophic Lateral Sclerosis}

Received Feb. 7, 2020; Accepted for publication Feb. 10, 2020; Published online Feb. 26, 2020

\section{ABSTRACT}

Objective: To determine safety and tolerability of two doses of ranolazine in patients with ALS and evaluate for preliminary evidence of drug-target engagement.

Background: Ranolazine, an FDA-approved drug for angina which inhibits the late $\mathrm{Na}+$ current and intracellular $\mathrm{Ca} 2+$ accumulation, may be neuroprotective in ALS and reduce cramp frequency by reducing neuronal hyperexcitability.

Design/Methods: Open-label dose-ascending study of ranolazine with 12 participants in two sequential cohorts: $500 \mathrm{mg}$ and 1,000 mg orally twice daily. Each has a two-week run-in period, four-week drug administration and six-week safety follow up. Primary outcome is the frequency of dose limiting toxicities and adverse events. Exploratory measures include cramp frequency and severity, fasciculation frequency (on ultrasound), cramp potential duration, ALS Functional Rating Scale-revised (ALSFRS-r), and forced vital capacity (FVC) compared from baseline to week six.

Results: The 10 currently enrolled patients (Cohort $1, \mathrm{n}=6$; Cohort $2, \mathrm{n}=4$ ) are mostly male ( $80 \%$ ), and middle aged (median 53 years). There were no serious adverse events. One subject in cohort 2 discontinued the drug due to constipation. The most frequent drug-related adverse event was gastrointestinal (38\%). Cramp frequency reduced by 44\% ( $\mathrm{p}=0.01)$ and severity decreased by 36\% ( $\mathrm{p}=0.002)$, with decreased awakening due to cramps and cramps interfering with daily activities or causing activity avoidance. Fasciculations, cramp potential duration, ALSFRS-r and FVC did not change.

Conclusion: Ranolazine was well tolerated in ALS up to 2,000 mg daily with gastrointestinal side effects being the most frequent. Ranolazine reduced cramp frequency and severity without significant effect on fasciculations, ALSFRS-r or FVC.

Funding Support: This work was supported by a CTSA grant from NCATS awarded to the University of Kansas for Frontiers: University of Kansas Clinical and Translational Science Institute (\#UL1TR002366). The contents are solely the responsibility of the authors and do not necessarily represent the official views of the NIH or NCATS. 


\title{
KANSAS JOURNAL of MEDICINE
}

\author{
Debra Sullivan, Ph.D., RD \\ Chair \\ Department of Dietetics and Nutrition \\ University of Kansas Medical Center \\ Frontiers Infrastructure Award Recipient
}

\section{Skin Carotenoid Measurement: A Biomarker for Dietary Fruit and Vegetable Intake}

Received Feb. 7, 2020; Accepted for publication Feb. 10, 2020; Published online Feb. 26, 2020

\begin{abstract}
Dietary intake of fruits and vegetables is consistently related to chronic disease risk. Unfortunately, measurement of fruit and vegetable intake is most often by self-report and thus, there are concerns about the validity of the data. The Veggie Meter (VM) uses Resonance Raman light scattering spectroscopy (RRS) to non-invasively measure skin carotenoid content. Skin carotenoids have recently been reported as a reproducible and valid biomarker for dietary intake of fruits and vegetables. In August of 2017, we purchased a VM and began using it in several studies. Due to demand, we were unable to accommodate all studies. Thus, in July 2018 Frontiers provided funds to purchase another VM. To date, we have used VM in seven completed pilot studies (six interventions and one observational study) and are using it in three ongoing studies. Specifically, the Frontiers-purchased machine is being used in two NIH-funded clinical trials. It is also used in community outreach events. The average age of individuals screened using this VM is 74.9 years and the VM score is $281.9 \pm 70.1$, indicating poor intake similar to the national average. Within 54 healthy older adults, VM scores correlated with intake of vegetables ( $2=0.2$, $\mathrm{p}$ $=0.001)$, fruits and vegetables $(\mathrm{r} 2=0.15, \mathrm{p}<0.01)$, and four individual carotenoids $(\mathrm{r} 2>0.18 \& \mathrm{p}<0.01$ for all $)$ derived from self-reported 3-day food records. The VM is quick, non-invasive, and easy to use. It provides an objective measure of fruit and vegetable intake and will continue to be incorporated into our nutrition clinical trials and observational studies.
\end{abstract}

Funding Support: This work was supported by a CTSA grant from NCATS awarded to the University of Kansas for Frontiers: University of Kansas Clinical and Translational Science Institute (\#UL1TR002366). The contents are solely the responsibility of the authors and do not necessarily represent the official views of the NIH or NCATS. 
Frontiers Mentored Career Development Scholar (KL2)

\title{
Cognitive Function and Relationships with Intervention Dropout, Adherence and Weight Loss
}

\author{
Received Feb. 7, 2020; Accepted for publication Feb. 10, 2020; Published online Feb. 26, 2020
}

\begin{abstract}
Objective: Greater cognitive function (CF) is associated with adherence to prescription medications, better program adherence and weight loss (WL) following bariatric surgery. The purpose of this study was to evaluate the association between baseline CF, intervention dropout, adherence and three-month WL.
\end{abstract}

Methods: One hundred and seven (Mean age $=40.9$ yrs.), overweight $/$ obese $(\mathrm{BMI}=35.6 \mathrm{~kg} / \mathrm{m} 2)$ men $(\mathrm{N}=17)$ and women $(\mathrm{N}=90)$ completed a three-month WL intervention. Participants were asked to attend weekly behavioral sessions, comply with a reduced calorie diet and complete 100 min of moderate intensity physical activity (PA)/wk. CF tasks including Flanker (attention), Stroop (Executive control) and working memory, body weight and cardiovascular fitness (covariate) were assessed at baseline and three-months. Session attendance, adherence to PA and diet prescriptions and number of off-diet episodes were recorded weekly.

Results: Results indicated that attention was positively correlated with session attendance $(p=0.016)$, adherence to the diet $(p<0.01)$ and PA ( $\mathrm{p}=0.023)$. Executive control was positively correlated with WL ( $\mathrm{p}=0.042)$. Working memory (two tasks) was also positively correlated with $\mathrm{WL}(\mathrm{p}=0.017$ and $\mathrm{p}=0.025)$. Analysis of variance (ANOVA) indicated that baseline attention $(\mathrm{p}=0.012)$ was positively related to WL and negatively associated with drop out $(\mathrm{p}<0.05)$. Hierarchical linear regression showed executive control $(\mathrm{p}=0.036, \mathrm{R} 2=0.054)$ and working memory $(\mathrm{p}=0.013, \mathrm{R} 2=0.073$ and $\mathrm{p}=0.017, \mathrm{R} 2=0.068)$ were associated with $\mathrm{WL}$ when controlling for fitness.

Significance: These results suggest that stronger baseline attention is associated with completion of a three-month WL intervention. Executive control and working memory are associated with amount of WL achieved. Additional, larger and longer trials to assess the role of baseline CF on WL and evaluating the impact of interventions designed to improve CF on WL are indicated.

Funding Support: This work was supported by a CTSA grant from NCATS awarded to the University of Kansas for Frontiers: University of Kansas Clinical and Translational Science Institute (\#ULITR002366). The contents are solely the responsibility of the authors and do not necessarily represent the official views of the NIH or NCATS. 


\title{
KANSAS JOURNAL of MEDICINE
}

\author{
Karen Wambach Ph.D., RN, IBCLC, FILCA, FAAN \\ Professor \\ School of Nursing \\ University of Kansas Medical Center
}

Frontiers Dr. Lauren S. Aaronson Trailblazer Recipient

\section{Adolescent Mothers' Early Breastfeeding Experiences}

Received Feb. 7, 2020; Accepted for publication Feb. 10, 2020; Published online Feb. 26, 2020

\begin{abstract}
Introduction: Adolescent mothers are less likely to initiate and sustain exclusive or partial breastfeeding. About $70 \%$ of mothers under age 20 initiate breastfeeding, compared to $80 \%$ of 20 - 29-year-old mothers. Duration of breastfeeding to six months and one year by young mothers is $28 \%$ and $18 \%$, compared to $48 \%$ and $28 \%$ in 20 - 29-year olds. Early breastfeeding experiences can adversely influence duration of breastfeeding. This study describes the early postpartum breastfeeding experiences among mothers 16 - 19 years of age.
\end{abstract}

Methods: This is a descriptive study within a larger pilot clinical trial. Four to eight weeks after giving birth, participants completed the Breastfeeding Experience Scale (BES), a 30-item measure which documents breastfeeding experiences, feeding patterns, and weaning. Severity of 18 early experiences are rated on a Likert type scale of $1-5$ ( 1 = "not at all" to $5=$ "unbearable"). Cronbach's alpha for the 18 -item summated scale of the BES was 0.80. Descriptive statistics characterized experiences for this in-progress study.

Results: Thirty breastfeeding participants, ages $16-19(\mathrm{X}=17.97$, SD = 0.96) completed the BES. Twenty-one $(70 \%)$ were breastfeeding at time of survey and $57 \%$ were doing so exclusively. The most frequently reported breastfeeding problems were engorgement and leaking which were rated mild to moderate in severity $(X=2.97, \mathrm{SD}=1.03)$. Nine mothers $(30 \%)$ weaned between 14 and 30 days after birth; engorgement, latching difficulty, and drop in milk supply were most common reasons.

Conclusions: Most teenage mothers in this sample maintained breastfeeding past one month after birth and reported few problems.

Funding Support: This work was supported by a CTSA grant from NCATS awarded to the University of Kansas for Frontiers: University of Kansas Clinical and Translational Science Institute (\#UL1TR002366). The contents are solely the responsibility of the authors and do not necessarily represent the official views of the NIH or NCATS. 
Yun Yan, M.D.

Pediatric Endocrinologist

Children's Mercy Kansas City

Institute for Advancing Medical Innovation (IAMI) Trailblazer Award Recipient

\author{
A Therapeutic Trial Validating the Physiologically Based Pharmacokinetic Simulation of the Dose-Exposure Relationship of \\ Metformin in Young Children with Insulin Resistance or Type 2 Diabetes Mellitus \\ Chelsea Cojocari, Ph.D., Rachel Frazier, RN, BSN, CCRC, Kelsee Halpin, M.D., Paul Toren, Ph.D., \\ J. Steven Leeder, Pharm.D., Ph.D., Yun Yan, M.D. \\ Received Feb. 7, 2020; Accepted for publication Feb. 10, 2020; Published online Feb. 26, 2020
}

\begin{abstract}
Introduction: Obesity affects $~ 14$ million children/adolescents in the U.S. (8.9\% of 2 - 5-year-olds; $17.5 \%$ of 6 - 11-year-olds) and increases the risk of developing type 2 diabetes (T2DM). Early metformin intervention may delay or halt progression to T2DM. However, clinical response to metformin in children with insulin resistance or T2DM is unpredictable. Additionally, no dosing recommendations exist for children $<10$ years. Preliminary data show that clinical response to metformin may depend on the amount of metformin present in the body over time (systemic exposure), with maximum response observed at exposures $=30 \mathrm{mg} / \mathrm{L}^{*} \mathrm{~h}$. Modelling and simulations can help determine individualized doses to achieve this exposure, ideally achieving favorable response for every patient. This study aims to prospectively validate simulations of the metformin dose-exposure relationship in young children.
\end{abstract}

Methods: A 24-hour pharmacokinetic study (measuring systemic exposure) of 500 mg metformin in children (6 - 12 years) with IR. Pharmacokinetic simulations including individual parameters (e.g., sex, age, weight) were conducted for each subject using Simcyp, a physiologically-based pharmacokinetic simulator.

Results: Two out of 12 subjects have completed the research study: Subject 1: Female, 12 years, $158.6 \mathrm{~cm}, 70.2 \mathrm{~kg}$, BMI: 97.44 percentile, HbAlC: 6.7\%; simulation-recommended dose: 1750 mg. Subject 2: Male, 8 years, 134.9 cm, 36.5 kg. BMI 95.58 percentile, HbAlC: 6.5\%; simulation-recommended dose: $1100 \mathrm{mg}$. Clinical pharmacokinetic data is not yet available.

Conclusion: Individualized doses of metformin may benefit young children with IR. Smaller/younger patients may require smaller doses of metformin than larger/older children; clinical pharmacokinetic data is needed to support simulation-guided individualized dosing.

Funding Support: This work was supported by a CTSA grant from NCATS awarded to the University of Kansas for Frontiers: University of Kansas Clinical and Translational Science Institute (\#UL1TR002366). The contents are solely the responsibility of the authors and do not necessarily represent the official views of the NIH or NCATS. 


\title{
KANSAS JOURNAL of MEDICINE
}

\author{
Yuxia (Lisa) Zhang, Ph.D. \\ Assistant Professor \\ Department of Pharmacology, Toxicology, and Therapeutics \\ University of Kansas Medical Center
}

Frontiers Clinical and Translational Pilot Research Grant Recipient

\author{
Targeting RNA-Binding Protein HuR in Human Liver Cancer \\ Priyanka Ghosh' ${ }^{1}$ Lin $\mathrm{He}^{1}$, Nancy Magee ${ }^{1}$, Forkan Ahamed ${ }^{1}$, Xiaoqing $\mathrm{Wu}^{2}$, Liang Xu², Yuxia Zhang ${ }^{1}$ \\ ${ }^{1}$ University of Kansas Medical Center, Department of Pharmacology, Toxicology \& Therapeutics, Kansas City, KS \\ ${ }^{2}$ University of Kansas, Department of Molecular Bioscience, Lawrence, KS \\ Received Feb. 7, 2020; Accepted for publication Feb. 10, 2020; Published online Feb. 26, 2020
}

\begin{abstract}
Introduction: Hepatocellular carcinoma (HCC) is one of the most common cancers worldwide and has one of the worst one-year survival rates of any cancers. Understanding molecular mechanisms leading to HCC and developing novel strategies for effective prevention and treatment are urgent medical needs. HCC arises exclusively on the background of chronic liver injury and inflammation. Thus, an attractive therapeutic approach is to target key genes that regulate both inflammation and oncogenic pathways in HCC. RNA-binding protein Hu Antigen $\mathrm{R}(\mathrm{HuR})$ is one such candidate that controls the translation of multiple genes involved in inflammation and oncogenic process. The objective of this study is to characterize HuR targets in human HCC and test HuR-targeted intervention for HCC treatment.
\end{abstract}

Methods: HuR knockout (HuR-KO) in HCC cell line MHCC97H cells was accomplished by CRISPR/Cas9 gene editing system. The transcriptome comparison identified 1,5l6 differentially-expressed genes between HuR-KO cells and wild type controls. The top pathways altered by HuR knockout were cell proliferation, hippo signaling, beta integrin, pluripotent stem cells, EGFR, WNT, and FAK-PI3K-Akt-mTOR. To test HuR-targeted intervention for HCC treatment in vivo, MHCC97H cells were subcutaneously injected into athymic nude mice to establish HCC xenografts. Mice were then treated with a novel synthetic HuR inhibitor KH-3 for four weeks.

Results and Conclusions: Strikingly, KH-3 treatment significantly inhibited the growth of HCC xenografts. Taken together, our study provides a foundation for understanding the role of HuR in HCC. The mechanistic insights elucidated from our study may pave the way for developing therapeutic strategies to specifically target HuR for HCC treatment.

Funding Support: This work was supported by a CTSA grant from NCATS awarded to the University of Kansas for Frontiers: University of Kansas Clinical and Translational Science Institute (\#UL1TR002366). The contents are solely the responsibility of the authors and do not necessarily represent the official views of the NIH or NCATS. 